\title{
Training vs. Coaching - über Aporien in der Professionalisierungsdiskussion
}

\author{
Thomas Webers ${ }^{1,2}$ iD $\cdot$ Robert Zickermann ${ }^{1}$ \\ Online publiziert: 6. November 2019 \\ (c) Der/die Autor(en) 2019
}

\section{Zusammenfassung}

Die Abgrenzungsdebatte um die Professionalisierung von Coaching ist unabgeschlossen und weiterhin notwendig. Coaching wird beispielsweise immer wieder mit Training verglichen. Die Entleihung des Begriffs Coaching aus der Welt des Sports ist ein Fakt, ob der Vergleich sinnvoll ist, eine offene Frage. Die Erforschung des Sprachgebrauchs in der Sportwissenschaft zeigt, dass trotz der steigenden Popularität Coaching auch im Sport ein unscharfer Begriff geblieben ist. In der Coaching-Literatur werden zur Begründung der Abgrenzung mehrere Konzepte bemüht. Die Analyse zeigt, dass diese u. a. Hilfe zur Selbsthilfe, Beratung ohne Ratschlag - einer kritischen Diskussion nicht standhalten. Welche Lehren können nun aus dieser Debatte für eine weitere Professionalisierung von Coaching gezogen werden?

Schlüsselwörter Professionalisierung · Domänen $\cdot$ Coaching $\cdot$ Training $\cdot$ Rolle $\cdot$ Dienstleistung

\section{Training Versus Coaching: Aporia in the Debate of Professionalization}

\begin{abstract}
The demarcation debate about the professionalization of coaching is unfinished and still necessary. One reason for this debate is the transfer of the term coaching from the world of sports. Coaching is often compared and even equated with training. But does coaching in sports mean the same as training or can differences be made? Despite the rising popularity, coaching in sports has remained a vague concept. So on the other hand in business coaching: The reference to concepts like helping people helping themselves or advice without advice are paradoxical and non-discriminating. What can be resumed by this debate for the professionalization of coaching?
\end{abstract}

Keywords Professionalization $\cdot$ Domains $\cdot$ Coaching $\cdot$ Training $\cdot$ Role $\cdot$ Professional Service

\section{Coaching vs. Training}

Der Gründungsmythos von Coaching basiert auf der Provenienz aus der Sport-Domäne (Greif et al. 2018). Umso erstaunlicher erscheinen Abgrenzungsbemühungen der Coaching-Branche - nicht nur zur Psychotherapie, Supervision, Beratung etc. - sondern eben auch in Richtung Training. Im Jahr 2015 veröffentlicht der ,,Roundtable der Coachingverbände“ ein von zehn Einzelverbänden getragenes Po-

Thomas Webers

coaching@thomas-webers.de

1 Hochschule Fresenius, Köln, Deutschland

2 FOM-Hochschule, Bonn, Deutschland sitionspapier „Profession: Coach“ (RTC 2015). Dort wird Coaching von Expertenberatung, Weiterbildung und Psychotherapie abgegrenzt. Rauen (2014) grenzt - wie schon Schreyögg (2012) für den „Deutschen Bundesverband Coaching“ (DBVC) - Coaching u. a. von Training ab. Aus einer Reihe seiner Argumente stechen diese hervor:

- Die Funktion von Training liege darin, gezielt bestimmte Verhaltensweisen zu entwickeln. Im Coaching hingegen gehe es häufig um Haltung und Werte, die konkretem Verhalten zugrunde liegen - es also bspw. sabotierten.

- Der Trainer sei eher sachorientierter Anleiter und Experte, der Coach eher beziehungsorientierter Zuhörer und Reflexionspartner.

- Die Trainingszielgruppe sei breit, die Inhalte von Trainings seien oft vorgegeben oder standardisiert (z.B. Ver- 
kaufstraining). Coaching wiederum fokussiere auf Führungskräfte, die in der Regel schon etliche Trainings absolviert hätten, nun aber individuell an ihrer Haltung arbeiten wollten.

Dieser Widerspruch zwischen Provenienz und Distanzierung ist erklärungsbedürftig. In diesem Beitrag wird die These vertreten, dass die Abgrenzungsbemühungen ihren Grund in einer nur teilweise gelungenen Marktpositionierungsstrategie haben. Denn es lassen sich nicht nur Unterschiede, sondern ebenfalls Gemeinsamkeiten finden, so dass sich keine trennscharfe Unique Selling Proposition (USP) benennen lässt. Die Abgrenzungsdiskussion erscheint bei näherer Betrachtung als oberflächlich, es fehlt eine differenzierte Bestandsaufnahme des Begriffsgebrauchs in der Domäne Sport. Daher stellen sich folgende Forschungsfragen:

- Werden die Begriffe Training und Coaching in der Sportwissenschaft synonym gebraucht?

- Wie wird eine Abgrenzung zwischen Training und Coaching in der Sportwissenschaft begründet?

- Welche sinnvollen Konsequenzen hat die Abgrenzung gegenüber Training für Coaching?

- Welche nützlichen Hinweise können für eine weitere Professionalisierung gegeben werden?

\section{Abgrenzungsdebatten als Teil der Professionalisierungsdiskussion}

Seit den 1980er-Jahren begleitet eine Abgrenzungsdebatte die Professionalisierung von Coaching (Schmidt-Lellek und Fietze 2018). Dies lässt sich als eine notwendige Entwicklung begreifen, denn auf dem Markt konkurrieren diverse Angebote miteinander. Wer sich nicht abgrenzen kann, wird sich langfristig nicht behaupten können. Kühl (2008) unterscheidet zwei Modelle der Marktregulierung:

- Professionalität im engeren Sinne: Akademisierung, Standardisierung, Regulierung. Professionelle unterwerfen sich den durch eine Profession festgelegten Standards (,klassische“ Professionsbildung durch vom Staat geschützte Institutionalisierung - der kontinentaleuropäische Weg).

- Professionalität im weiteren Sinne: Professionelle entwickeln weitgehend individuell und möglichst wenig durch externe Normen eingeschränkt Vorstellungen von Professionalität auf der Basis einer Selbstverpflichtung (,,moderne“, marktgetriebene Professionalisierung - der angloamerikanische Weg).

$\mathrm{Ob}$ Coaching als Profession zu bezeichnen ist, ist aus kontinentaleuropäischer Perspektive betrachtet an einer Professionalisierung im engeren Sinne zu messen. Jenseits eines Erfolgs am Markt wäre dies an - Schmidt-Lellek (2006) nennt insgesamt zehn - Merkmalen zu überprüfen:

- Verantwortungsvolle Arbeit am Menschen: Personenbezogene, immaterielle Dienstleistung.

- Mitwirkung des Klienten: Koproduktion zwischen Professionellen und Klienten.

- Eine Lizenz: Auf einer theoretisch fundierten (akademischen) Ausbildung basierend.

- Ein gesellschaftliches Mandat: Monopolisierte Stellung zwischen Staat und Markt.

- Applikation des Wissens: Eine individualisierte „Übersetzungsarbeit" von Fachwissen auf den Einzelfall.

- Ethik-Kodex: Dessen Verletzung kann zu einem Ausschluss aus der Profession führen.

- Berufsverband: Eine selbstverwaltete Organisation der Professionsangehörigen.

- Fachautorität und Prestige: Exklusive Entscheidungskompetenzen (Handlungsmonopol).

- Spezifika: Berufliche Rollenmuster, Karrieregänge, Statuspassagen.

- Finanzielle Unabhängigkeit: Verbeamtung oder Gebührenordnungen.

Diese Merkmale basieren wiederum auf der berufssoziologischen Unterscheidung von Arbeit, Beruf und Profession (Birgmeier 2006):

- Arbeit meint grundsätzlich diverse Tätigkeiten (auch ehrenamtliche) Arbeit, Haus- und Freizeitarbeit.

- Beruf beschreibt eine Tätigkeit, die in einem gesellschaftlich organisierten Kontext erbracht und auf dem Arbeitsmarkt gegen Bezahlung geleistet wird.

- Profession meint eine spezielle Ausprägung gehobener beruflicher Tätigkeit, die mit einem Expertenstatus, einem Berufsethos, einer berufsständischen Organisation sowie mit gewissen Entscheidungskompetenzen (Handlungsmonopol) verbunden ist.

Das Projekt einer Professionalisierung des Coachings zielt damit auf den höchsten Status. Als klassische Professionelle gelten u.a. Juristen, Ärzte, Pfarrer, Lehrer (Schmidt-Lellek 2006). Der Staat hält traditionell seine schützende Hand über diese Professionen, ermöglicht ihnen eine Teilautonomie in Selbstverwaltung, die allerdings auch eine Kostenseite hat - beispielhaft seien hier die Pflichtkammermitgliedschaft, Kassenzulassung etc. genannt. Wer also die Professionalisierung von Coaching nach diesem Modell anstrebt, benötigt folglich eine gesetzliche Grundlage, Institutionen, geregelte Karrierewege und so weiter. Mehr Staat oder mehr Markt? Die Diskussion dieser Richtungsentscheidung begleitet die Entwicklung von Coaching - wie schon gesagt - von Beginn an.

„Ob sich ein eher an breiter Professionalisierung oder ein eher an enger Professionsbildung orientierter Ansatz in der 
personenzentrierten Beratung durchsetzen wird, ist zurzeit nicht abzusehen," so Kühl (2008, S. 282) seinerzeit. Fietze (2015) sieht die Entwicklung ein knappes Jahrzehnt später insgesamt positiv, wenn auch immer noch widersprüchlich:

- Professionelle Expertise: Der anfänglich eher feuilletonistische Diskurs transformierte sich inzwischen in einen anspruchsvollen wissenschaftlichen. Praxisfelder differenzieren sich aus. Es lässt sich dabei eine Ausrichtung auf übergreifende Fragestellungen, eine Multidisziplinarität und Methodenpluralität beobachten.

- Positionierung: Dessen ungeachtet bleibe Coaching ein unscharfer Containerbegriff, der sich nicht leicht gegen Nachbardisziplinen abgrenzen lasse. Der Streit um Definitionen diene auch immer der (Selbst-)Positionierung auf dem Markt.

- Qualitätsmanagement: Wenn auch die Professionsbildung im klassischen Sinne nicht gelungen sei, so seien doch Fortschritte in der Professionalisierung zu verzeichnen: Standards, Zertifizierungen, Akkreditierungen, die Beobachtung der Qualitätsentwicklung durch den Verbraucherschutz und die mediale Öffentlichkeit.

- Korporatistische Selbstverwaltung: Es haben sich etliche Fach- und Berufsverbände etabliert, deren Mitgliederzahlen und Binnendifferenzierungen stiegen. Mit dem Roundtable der deutschsprachigen Coachingverbände (RTC) ist nun auch eine überverbandliche Plattform entstanden. Ein Interesse des Staates an einem CoachGesetz in Deutschland ist allerdings nicht festzustellen. Voraussetzung eines solchen Gesetzes wäre eine staatlich anerkannte oder wissenschaftlich fundierte Ausbildung zum Coach (Beruf). Coaching ist aber (nur) eine Weiterbildung - auch wenn die beliebte Etikettierung als Coaching-Ausbildung immer wieder anderes suggeriert - und diese ist Privatsache. Die Regelung der Gewerbeordnung in Österreich gilt als gescheitert, da Coaching und Supervision zwar als freies Gewerbe gelten, sich damit aber ein gesetzlicher Widerspruch zu den stark reglementierten Tätigkeiten der Lebens- und Sozialberatung, der Unternehmensberatung sowie der Psychotherapie ergibt. Eine europaweite Erhebung konstatiert, „,dass SupervisorInnen \& Coaches in einem mehrheitlich gesetzlich nicht geregelten Gewerbe tätig sind" (Anger und Schober 2014, S. 50). Daher sprechen die Autoren auch von einer rechtlichen Grauzone.

„Obwohl also die Professionalisierung von Coaching bemerkenswerte Fortschritte gemacht hat, genügt Coaching dem idealtypischen Kriterienkatalog des klassischen Professionsmodells nicht, weil ein klar zugeschnittener Problemtypus und ein klar abgegrenzter Zuständigkeitsanspruch fehlen“ (Fietze 2015, S. 11 f.). Allerdings konstatiert die Autorin eine andere Seite dieser unabgeschlossenen Entwicklung, die diese in einem freundlicheren Licht er- scheinen lässt. Denn der Modernisierungsprozess hat den Status der staatlich protegierten, klassischen Professionen zunehmend relativiert:

- Liberalisierung: Der Staat hat sich aus etlichen Bereichen zurückgezogen (Deregulierung), zunehmend regelt der Markt das Angebot.

- Informationsexplosion: Das exklusive Machtmonopol der Experten ist im Internetzeitalter nur noch relativ, selbst Expertenwissen ist heute leicht verfügbar.

- Kultureller Wandel: Statt standardisiertem Fachwissen wird heute Flexibilität in Denken und Handeln statt Dogmatik als professionelle Kompetenz erwartet.

Die Welt hat sich deshalb auch für die klassischen, privilegierten Professionen verändert:

- Die Kirchen verlieren Mitglieder und Glaubwürdigkeit, zahlreiche neue religiöse Bewegungen sind auf dem Markt. Die herausragende und sakrosankte Stellung von Priestern, Geistlichen und Pfarrern, die noch vor Jahrzehnten galt, ist heute - auch schwer beschädigt durch zahlreiche Missbrauchsskandale - nicht mehr gegeben.

- Ärzte müssen mit allerlei (finanziellen) Anreizen von der Ausreise in lukrativere Staaten oder dem Anheuern bei der Pharmaindustrie abgehalten oder von den Städten aufs Land gelockt werden, zugleich ermöglicht man ihnen die für sie lukrativere Behandlung von Privatpatienten.

- Der unregulierte Zugang zur Juristenausbildung hat unter diesen einen starken Wettbewerb entfacht. Auch für diese Profession eröffnen sich lukrative Arbeitsfelder in der Privatwirtschaft.

- Die Verbeamtung als Lehrer ist angesichts verkrusteter Strukturen in Schulen, ideologisch polarisierten, politischen Grabenkämpfen, teilweise aggressiven Kindern und sog. Helikopter-Eltern, Berichten über hohe Burnout-Quoten unter Lehrern sowie ausgeprägten Lehrermangel bspw. in Grundschulen für viele nicht mehr attraktiv.

Fietze (2015) diagnostiziert einen allgemeinen Entberuflichungsprozess (De-Professionalisierung) in der Moderne. Soll es für die Coaching-Branche sinnvoll sein, hinter dem Ideal alter Professionen herzulaufen, wenn sich diese gerade auflösen? Wäre nicht die Orientierung am Modell der Professionalisierung im weiteren Sinne - also am Markt zweckdienlicher? Denn interdisziplinäre Zusammenarbeit, multiprofessionelle Perspektiven, Relativierung alter Gewissheiten prägen den aktuellen gesellschaftlichen Diskurs.

Schulz konstatiert, „dass das, was als ,Coaching bezeichnet wird, bei genauerem Hinsehen sehr verschiedene soziale Interaktionen sind. Mit Coaching werden unterschiedliche Formen der personenzentrierten Personalentwicklung, Mitarbeitergespräche im Rahmen der Personal- 
führung, eine Unterstützung von Verkaufs- und Akquisetrainings, Gruppendynamische Seminare sowie nicht näher definierte Beratungsformate zusammengefasst" (Schulz 2016, S. 2f.). In dieser Gemengelage ist die Positionierung, wie Fietze konstatiert, eine Strategie unter anderen, um eine Unique Selling Proposition (USP) gegenüber Wettbewerbern zu behaupten. Doch wovon sich gegenüber „Training“ aus Coaching-Sicht abgegrenzt werden soll, bleibt meist unklar. Daher lohnt der genauere Blick auf die Sportdomäne.

\section{Training und Coaching in der Domäne Sport}

\subsection{Sportwissenschaftliche Konzeption}

Im deutschsprachigen Standardwerk der Sportwissenschaft, „Optimales Training“, wird auch in der aktuellen Auflage (Weineck 2019) konstatiert, dass die Begriffe Trainer und Coach in der Praxis synonym gebraucht würden, allerdings Unterschiedliches adressierten:

- Trainer seien für die Ausbildung, die technische Schulung, das Einüben zuständig. Training sei sachbezogen (Vorbereitung auf den Wettkampf).

- Aufgaben von Coaches sei hingegen die Beratung und psychologische Führung. Coaching sei personenbezogen (Begleitung im Wettkampf).

Hier wird ein Stufenmodell postuliert, einer Junior-/ Senior-Positionierung vergleichbar: Der Coach wird hierarchisch über den Trainer gestellt, wenngleich Coaching als Teilbereich der Trainingstätigkeit weiterhin reklamiert wird. Im Sinne eines klassisch-cartesianischen Körper/ Geist-Dualismus bereitet der Trainer den Körper auf den Wettkampf vor. Der Coach kümmert sich im Wettkampf um den Geist. Er vermittelt Sicherheit, Mut und Risikobereitschaft, aber fördert auch Konzentration sowie, im Falle eines Mannschaftssports, Verantwortungsbereitschaft fürs Team. Zudem interveniert der Coach aktiv im Wettkampf: Er steuert den Personaleinsatz, die Pausengestaltung und gibt taktische Anweisungen.

Es ist folglich Personalführung, was der Coach leistet (der Coach als Führungskraft). Das gilt nicht nur für den Einzelathleten, sondern zeigt sich im Mannschaftssport noch viel offensichtlicher, wenn der Trainer - wie im Fußball - in seiner „Coaching-Zone“ am Spielfeldrand operiert. Als Führungskraft ist er dort, wenn nicht der „12. Mann“, diese Rolle hat der Volksmund dem Publikum zugedacht, dann so etwas wie der „13. Mann“. Oder seine Funktion wird noch viel gewichtiger eingeschätzt. Denn als genuine Aufgabe des Trainers wird die Strategieentwicklung bezeichnet (Hohmann et al. 2014). Er ist der Steuermann, der „Kopf“.
Damit ist der Trainer auch Teil des Teams. Dies lässt sich insbesondere an den sog. Coaching-Rules aufzeigen. Je nach Sportart darf der Trainer den Sportler im laufenden Wettkampf über Details wie Rangplatz, Verfolger, Beschaffenheit des Sportareals etc. informieren, so dass sich der Athlet selbst besser steuern kann (Bechthold 2014). Oder er darf taktische Anweisungen geben und sogar ins Spiel eingreifen (z. B. Auswechslung, Timeouts z. B. im Handball oder Basketball). Dabei kann er verschiedenartige Ziele verfolgen wie z. B. den Spielfluss der gegnerischen Mannschaft unterbinden oder die eigene Spieltaktik an neue Gegebenheiten (z. B. Verletzung eines Führungsspielers) anpassen. Beim Damentennisfinale der US Open 2018 im New Yorker Arthur Ashe Stadion kam es beispielsweise zum Eklat: Schiedsrichter Carlos Ramos verwarnte Tennisstar Serena Williams wegen illegalen Coachings. Offensichtlich hatte Trainer Patrick Mouratoglou mit Handzeichen versucht, Einfluss auf das Spiel der Athletin zu nehmen, was nach den Regeln des Tennis-Weltverbandes ITF nicht erlaubt ist. Williams bestritt dies, beleidigte den Schiedsrichter und zertrümmerte ihren Schläger.

Sehr deutlich zeigt sich diese Rolle des Trainers als Führungskraft an einem weiteren Aspekt, der Rolle als „Außenminister“: Die Medien interviewen den Trainer bspw. zum Wettkampf oder Spielverlauf. Sie machen ihn auch verantwortlich für das Ergebnis. Wenn ein neuer Trainer engagiert wird, verändert sich folglich das Spiel. Es kann sogar Ausdruck einer ,psychologischen Kriegsführung“ sein, wenn plötzlich die „Trainerlegende“ die Arena betritt. Im Tennis erscheinen mit ehemaligen Ausnahmespielern wie Andre Agassi, Ivan Lendl und Boris Becker immer häufiger sogenannte „Super-Coaches“ auf der Bühne, welche ihre Schützlinge zu Höchstleistungen anspornen (sollen). Alleine deren Anwesenheit scheint zu wirken - nicht nur auf das Publikum.

Dass sich dieser Körper/Geist-Dualismus in der Sportwissenschaft weiterhin behauptet, verwundert nicht, wenn man mit Schlicht (2008) die Genese des Fachs aus der Praxeologie beschreibt. In dieser steht traditionell die Physis im Fokus. Psychologie sei für das Fach lediglich eine Bezugs-

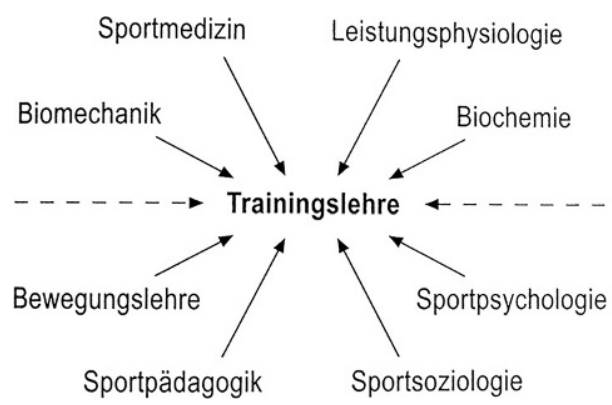

Abb. 1 Trainingslehre als interdisziplinärer Fachbereich. (Weineck 2019, S. 19) 
wissenschaft unter anderen (s. Abb. 1). Spiegelbildlich verhalte es sich allerdings mit der akademischen Psychologie. Für sie sei Sport überwiegend ein (Nischen-)Anwendungsfeld des eigenen Grundlagenwissens aus den Bereichen Allgemeine, Differenzielle und Sozialpsychologie. Schlicht berichtet den hegemonialen Streit detailliert und schlägt eine dritte Position vor, nicht ohne mit einem Seitenhieb festzustellen, ,dass Lehrende und Forschende der Sportpsychologie nahezu ausnahmslos an Instituten für Sportwissenschaft tätig sind" (Schlicht 2008, S. 34). Doch das zweibändige Werk „Sportpsychologie“ innerhalb der „Enzyklopädie der Psychologie“", zu dem der Beitrag von Schlicht die Einführung bildet, behandelt das Thema Sportpsychologie trotz allem klassisch, an den Grundlagenfächern der Psychologie (Bd. 1) und an Zielgruppen (Bd. 2) orientiert. So verfahren auch weiterhin diverse jüngere sportpsychologische Lehrbücher (Brand 2010; Baumann 2015). Positiv kann immerhin festgehalten werden, dass der Wissensfundus, der die Brücke zwischen dem Trainer und dem Coach in der sportwissenschaftlichen Konzeption bilden soll und kann, prinzipiell vorliegt. Was jedoch zumeist vermisst wird, ist die grundsätzliche kritische Rollenklärung der Trainerfunktion.

\subsection{Sportpsychologische Konzeptionen}

Die Sportpsychologie hat sich zuletzt von diesen klassischen, sportwissenschaftlichen Standpunkten emanzipiert, wie die Werke von Alfermann und Stoll (2012) sowie Hänsel et al. (2016) zeigen. Beide Autorengruppen referieren die Forschungen zum Trainerverhalten, die seit den 1970er Jahren in den USA betrieben wurden. Smith und Smoll (1996) hatten das Coaching Behavior Assessment System (CBAS) mit zwölf Verhaltenskategorien vorgelegt. Als übergeordnete Verhaltenskategorien bezeichnet das CBAS Unterstützung, Instruktion und Bestrafung. Chelladurai und Riemer (1998) entwickeln diesen Ansatz zum multidimensionalen Konzept Leadership Scale for Sports (LSS) weiter. Drei der fünf Dimensionen des LSS beziehen sich auf die Trainer-Athlet-Interaktion (Instruktion, Feedback, Unterstützung), zwei auf das Ausmaß der Mitbestimmung durch die Athleten (demokratisches bzw. autokratisches Verhalten).

Diesen Fortschritt würdigend gehen Alfermann und Stoll einen entscheidenden Schritt weiter, indem sie postulieren: „Es scheint an der Zeit, die Ansätze von Smoll und Smith und von Chelladurai zu erweitern um Merkmale der Trainer-Athlet-Beziehung" (Alfermann und Stoll 2012, S. 231). Sie kritisieren die gängige sportpsychologische Beratung und Betreuung von Athleten aus sozialpsychologischer Sicht als einseitig auf den Athleten fokussiert. Daher fordern sie die Erweiterung der Blickrichtung auf den Trainer und insgesamt darauf, dass beide - nicht nur im Mann- schaftssport - ein Team darstellen. Sie rekurrieren damit auf eine Studie von Jowett und Cockerill (2003). Die soziale und emotionale Beziehung zwischen Trainer und Athlet sehen diese Forscher durch drei Faktoren bestimmt:

- Closeness - Zuneigung, Vertrauen, Respekt

- Complementarity - Passung

- Co-orientation - ein gemeinsamer Bezugsrahmen von Zielen, Werten und Erwartungen

Damit wird die klassisch sportwissenschaftliche Konzeption - Schnabel et al. (2011) warnen vor einer gemeinsamen Augenhöhe von Trainer und Sportler - begründet infrage gestellt. Für Killing ist Training schon eine Dekade zuvor primär eine pädagogische Funktion, die sich vom Führer/ Lehrer einer Athletengruppe mit der Zeit zum Betreuer/ Partner entwickelt. „Leistung ist nicht das alleinige Produkt des Athleten, sondern vielmehr eine Gemeinschaftsproduktion von Athlet, Trainer und flankierenden Rollenträgern. Der Athlet ist der physische Träger der Leistung" (Killing 2002, S. 50). Auch der Nestor der deutschen Sportpsychologie, Hans Eberspächer, argumentiert in dieselbe Richtung (Eberspächer 2005). Dieser Paradigmenwechsel hin zu einer interaktionalen Perspektive lässt sich analog in der Geschichte der Führungspsychologie (Weibler 2013) verfolgen.

\subsection{Die internationale sportwissenschaftliche Diskussion}

Griffo et al. (2019) weisen international alleine 612 wissenschaftliche Zeitschriftenbeiträge mit Bezug zum Sport-Coaching für die Dekade 2005-15 nach - Veröffentlichungstendenz: steigend. Doch Cushion und Lyle bewerten die Lage ambivalent: Trotz der steigenden Popularität sei Coaching ein unscharfer Begriff geblieben. Unstrittig sei der Zweck der Performanz und, dass Coaching ein Beziehungsgeschehen sei. Versuche, dies weiter zu konkretisieren, zeigten jedoch, dass es sich eher um einen Gattungsbegriff handele, der je nachdem verschiedene Rollen, Domänen, Funktionen sowie Kompetenzen adressieren könne, letztlich aber schwammig bliebe: „Coaching is ill-defined and under theorized" (Cushion und Lyle 2016, S. 128). Als einen Grund für diese Befundlage machen die Autoren das herrschende positivistisch-reduktionistische Forschungsparadigma aus. Man zerlege soziale Praxis in seine Einzelteile, könne im Anschluss aus diesen aber keine tragfähigen ganzheitlichen Aussagen synthetisieren. In diesem Zusammenhang werten sie den Trend zur qualitativen Forschung in den letzten Jahren als der Komplexität von Coaching angemessener.

In dieselbe Richtung argumentiert North (2017). Er destilliert sechs Gemeinsamkeiten diverser Forschungsansätze für einen multidisziplinären Approach heraus. Sport-Coaching 
- hat Tiefgang - es als mechanische Aktivität zu charakterisieren, führe in die Irre: „A great deal that influences coaching practice is hidden/tacit“" (North 2017, S. 97);

- ist kontextgebunden - es ist eine Kooperation, die sozial und kulturell vermittelt ist, es kann davon nicht isoliert werden;

- ist zielorientiert - aber nicht monomanisch zu verstehen, Ziele sind zumeist mehrdimensional und verändern sich mit der Zeit;

- ist ressourcenorientiert - das Selbstmanagement individueller und sozialer/kontextueller Ressourcen steht im Fokus;

- ist sinnorientiert - es fördert strategisch die Reflexion von Einstellungen, Plänen und Entscheidungen;

- ist handlungsorientiert - situative Flexibilität im Verhalten und Lernbereitschaft wird angezielt.

\subsection{Der Trainer im Sport - ein Zwischenfazit}

Zusammenfassend betrachtet lassen sich einige zentrale Implikationen der in der Sportdomäne vertretenen Positionen herausarbeiten:

Menschenbild In der klassisch sportwissenschaftlichen Sichtweise wird der Athlet simplifizierend in Körper und Geist aufgespalten (Weineck 2019) und die Zuständigkeit für diese Domänen wird jeweils an die Trainer- bzw. Coach-Rolle delegiert. Diese Position ist wissenschaftlich längst überholt und dysfunktional - wie vor allem moderne neurobiologische Erkenntnisse (Roth und Ryba 2016) zeigen. Training im Sport, man wird das kaum anders bilanzieren können, muss auf den ganzen Menschen zielen. Dies ist in der Sportwissenschaft auch keine allzu neue Erkenntnis mehr: Schon im Jahr 2010 erschien die Zeitschrift für Sportpsychologie mit dem Schwerpunkt „Embodiment“ (4/2010).

Trainingsdesign Wenn die klassisch sportwissenschaftliche Sichtweise das Training vom Coaching unterscheidet, entsteht der Eindruck, die Trainingsmethodik (Kraft, Ausdauer, Schnelligkeit) stehe objektiv und unabhängig vom Athleten als One Best Way im Vordergrund. Wie Hottenrott (2017) jedoch zeigt, darf keinesfalls davon ausgegangen werden, dass die Trainingstätigkeit einer weitgehend standardisierten Massenware ähnelt. Im Gegenteil: Bei Training handelt es sich um einen hoch adaptiven und individuellen Prozess.

Didaktik Neben einer erzieherischen Funktion (Instruktion, Feedback, Unterstützung), Schnabel et al. (2011) sprechen dezidiert von notwendiger ,,autoritativer“ Führung, geht es den klassisch orientierten Sportwissenschaftlern ,,psycholo- gisch“ um die Emotionsregulation (anfeuern vs. dämpfen). Dies lässt sich als Instruktionsdidaktik (Arnold 2012) zusammenfassen: Wissen wird einseitig vom Lehrer zum Lerner transportiert (Nürnberger Trichter). Lernen wird als rezeptiver Vorgang aufgefasst. Der Lehrer ist nicht nur der Experte für das Wissen, sondern er steuert auch den Lernprozess und kontrolliert die -ergebnisse (Input-Orientierung). Dem Athleten wird eine eher passive Rolle zugedacht. Diese Sichtweise schließt an eine hierarchische Beziehungsdefinition an.

In modernen didaktischen Ansätzen wird von einem interaktiven Lerngeschehen ausgegangen. Im Modus der Ermöglichungsdidaktik (Arnold 2012) gibt der Lehrer Anregungen, bietet Unterstützung und Beratung an. Die Relation zum Athleten wird ganzheitlich und ,auf Augenhöhe“ gestaltet. Der Athlet eignet sich Wissen und Fertigkeiten individuell und aktiv explorierend an (Outcome-Orientierung).

Rollenkonzept Die Relation des Trainers zum Athleten wird klassisch hierarchisch beschrieben. Schnabel et al. (2011) leiten den Begriff Training aus dem Lateinischen ab (trahere: Das Herausziehen der Pferde aus dem Stall). Der Trainer als Reiter, das Pferd als „Sportgerät"? Sicher eine abschätzige, Interpretation, wenn man sie auf das Pferd oder erst recht auf den Menschen überträgt. Aber eine potenziell mögliche, technokratische Lesart. Man sollte diese Perspektive und die damit verbundene Gefahr der Instrumentalisierung der Athleten nicht aus dem Blick verlieren, sie bleibt eine latente Gefahr. Erinnert werden soll daher auch - neben dem Hinweis auf „Big Business“ und Doping-Kriminalität - daran, dass Kinder und Jugendliche im Sport-Kontext immer wieder Opfer sexueller Gewalt werden.

Die sportpsychologisch geprägte Konzeption fokussiert stark auf die sog. Augenhöhe der Interaktion zwischen Trainer und Athleten (Alfermann und Stoll 2012). Daher kann auch die Unterscheidung zwischen Trainer und Coach nicht sachlich begründet werden. Die Autoren kritisieren das Junior-/Senior-Modell (Trainer/Coach) als einseitig. Der Trainer übernimmt allerdings die Rolle einer Führungskraft.

Es lässt sich mithin konstatieren, dass in der Sportwissenschaft die Begriffe Training und Coaching weitgehend synonym verwandt werden, dass sich im Sprachgebrauch aber zwei unterschiedliche Auffassungen ausmachen lassen:

- Eine klassisch hierarchische Sichtweise: Der Coach steht über dem Trainer. Während der Coach als Führungskraft (zuständig für die Psyche) konzipiert wird, wird der Trainer als Gehilfe betrachtet, der für die Physis zuständig ist. 
- Eine moderne interaktive Sichtweise: Coach und Trainer werden nicht unterschieden, die Begriffe werden synonym und integral verwandt.

Ob der Sprachgebrauch jenseits der Sportwissenschaft, in der Sportpraxis oder in Massenmedien, ebenfalls synonym erfolgt, wäre empirisch zu untersuchen.

\subsection{Coaching: Ein Vergleich mit Training}

Auf dem Hintergrund der Ausführungen erscheint die Abgrenzung des Coachings vom Training - wie sie u. a. Rauen (2014) vornimmt - obsolet. Die postulierten Unterschiede lassen sich nur ausmachen, wenn man ausschließlich auf die klassische sportwissenschaftliche Sichtweise rekurriert. Deren Implikationen werden von der modernen, insbesondere sportpsychologischen Trainingsforschung jedoch nicht geteilt. Es ist sinnvollerweise von einer breiten Palette von Sportarten, athletischen Zielgruppen, Trainern sowie institutionellen Strukturen auszugehen, so dass von unterschiedlichen Konstellationen - durchaus auch im Sinne von Kontinua - auszugehen ist. Daher erscheint die von Rauen vorgetragene digitale Abgrenzung unpassend, weil einseitig überzeichnet. Alle von ihm angeführten Argumente können leicht entkräftet werden:

- Die Funktion von Training kann sich nicht darin erschöpfen, gezielt bestimmte Verhaltensweisen zu entwickeln. Auch an Haltung und Werten muss gearbeitet werden, der Ansatz im Sport sollte ein ganzheitlicher sein.

- Der Trainer sollte daher mehr als ein sachorientierter Anleiter und Experte sein, gleichfalls sollte er ein beziehungsorientierter Zuhörer und Reflexionspartner sein.

- Die Trainingszielgruppe mag breit und divers sein, die Inhalte von Trainings sind aber mitnichten standardisiert. Training ist ein hochadaptives Geschehen. Wenn sich Coaching auf Führungskräfte fokussiert, ist das eine selbstgewählte, selektive Setzung, die in der CoachingSzene übrigens auch nicht exklusiv von allen Stakeholdern geteilt wird (ICF o.J.).

\section{Zur Positionierung von Business-Coaching}

An dieser Stelle könnte man die Diskussion - auch mit dem Hinweis darauf, dass bislang keine verbindliche Definition von Coaching (Ives 2008) existiert - beenden. Wären da nicht noch interessante offene konzeptionelle Fragen.

Trainer sind im Sport häufig Führungskräfte. Sie sind Teil des Teams, Teil des gemeinsamen Sportunternehmens. Dies gilt nicht nur für zahlreiche Mannschaftssportarten, sondern ebenfalls für Einzelsportarten. In dieser Rolle fungieren Trainer gleichfalls als „Außenminister“ oder als Pressesprecher. Im Coaching tritt der Coach jedoch öffent- lich selten in Erscheinung. Intern oder im kleinen Kreis von Investoren mag es eine wichtige Information sein, dass die Top-Führungskraft von einem namhaften Coach begleitet wird, extern wird dies in der Regel nicht kommuniziert. Diskretion ist hier einerseits die Maxime. Andererseits möchte man Top-Führungskräfte auch nicht der Gefahr aussetzen, geschwächt zu wirken (Defizitmodell). Das Image einer Führungskraft im Business unterscheidet sich in dieser Hinsicht gravierend von dem des Sportlers: Während ein Voodoo-Faktor (bekreuzigen, Rasen küssen etc.) im Sport (Spiel) akzeptiert wird, passt dieser nicht zum seriösen Business (Ernst) - auch wenn sich hier gelegentlich Ausnahmen finden lassen: Auch im Sport wird unter Umständen viel Geld verdient und in der BusinessWeiterbildung finden sich gelegentlich unprofessionelle Auswüchse wie das Laufen über glühende Kohlen.

Zweitens wird Sporttrainern vielfach auch eine pädagogische Funktion zugesprochen. Klassischerweise erfolgt die Beschreibung der Rolle mittels der Instruktionsdidaktik (Lehrer vs. Schüler).

Beide Funktionen werden von zahlreichen Autoren sowie Coaching-Verbänden zur Abgrenzung aktiv aufgegriffen, dezidiert abgelehnt und somit zur Positionierung genutzt. Das sog. Funktionspendel (Wolff 2012), dessen sich der Deutsche Bundesverband Coaching (DBVC) bedient (s. Abb. 2), definiert drei Bereiche analog einer Ampelnavigation: Einen grünen Bereich (Potenzial-Aktivierung), einen gelben (Achtung!) sowie einen roten Bereich (Therapeut bzw. Schattenmanager). Im grünen Bereich soll es - auf Augenhöhe - um Klären, Reflektieren, Realitätsabgleich, Inspirieren, Umsetzen, Auswerten gehen. Nach dieser Vorstellung agiert der Sport-Trainer als Pädagoge, aber auch als Führungskraft, im sog. (roten) No-go-Bereich, da hier die gemeinsame Augenhöhe zugunsten einer hierarchischen Beziehung verlassen wird. Coaching sei ergebnisoffen, Führung nie, argumentiert Leder (2007). Der BusinessCoach, der in die Experten- oder sogar Managementfunktion gehe oder die Pädagogen- oder Therapeutenfunktion ausübe, beginge nach dieser Konzeption einen Kunstfehler. So offenbaren sich nun diskussionswürdige Unterschiede zwischen Business (Coaching) und Sport (Training).

Die Rekonstruktion dieser Argumentationsfigur verweist auf zwei Konzepte, die die Abgrenzung von Coaching gegenüber Training erklären und legitimieren sollen:

- Instruktions- vs. Ermöglichungsdidaktik sowie

- Experten- vs. Prozessberatung

\subsection{Instruktions- vs. Ermöglichungsdidaktik}

Schaper und Sonntag unterscheiden „zwei didaktische Grundorientierungen: die ,traditionelle ${ }^{`}$ Auffassung, die durch ein Primat der Instruktion geprägt ist und die ,kon- 
Abb. 2 Das sog. Funktionspendel. (Wolff 2012, S. 23)

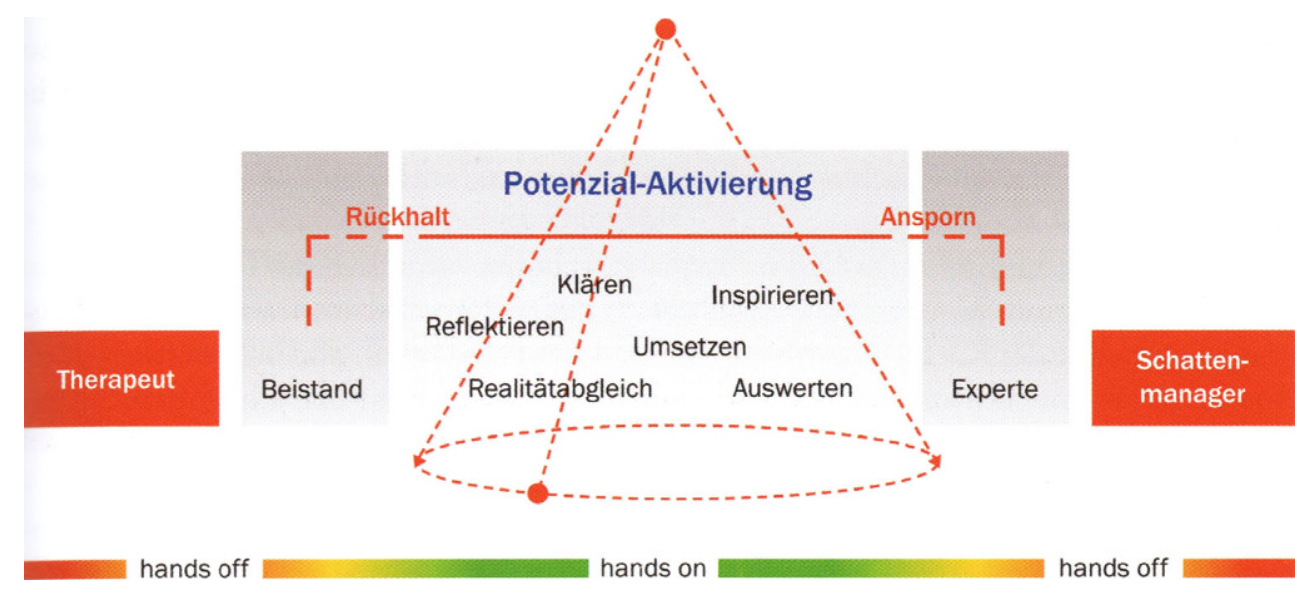

struktivistische" Auffassung, die durch besondere Annahmen über den Wissenserwerb beim Lernen gekennzeichnet ist" (Schaper und Sonntag 2007, S. $604 \mathrm{f}$.):

- Lernen durch Instruktion: Wissen wird vom Lehrer zum Lerner transportiert. Lernen wird als rezeptiver Vorgang aufgefasst. Der Lehrer ist nicht nur der Experte für das Wissen, sondern er steuert auch den Lernprozess und kontrolliert die -ergebnisse (Input-Orientierung). Kritisiert wird an dieser Auffassung die passive Rolle des Lerners (unklare Motivation und Selbstverantwortung) sowie die Vorstellung eines Lernens auf Vorrat.

- Lernen durch Ermöglichung: Menschen sind ,lernfähig, aber unbelehrbar“ (Arnold 2012, S. 46). Der Lehrer gestaltet deshalb realitätsnahe Lernumgebungen für den Lerner. Er gibt Anregungen, bietet Unterstützung und Beratung an. Der Lerner eignet sich Wissen und Fertigkeiten aktiv explorierend, situativ als Konstruktionsleistung an (Outcome-Orientierung). Kritisiert wird an dieser Auffassung, dass Lerner unterschiedlich gut auf Selbstaktivierung ansprechen, manche damit auch überfordert sind.

Diese Unterscheidung mag eine hohe Augenscheinvalidität besitzen, sie lässt sich aber kaum sinnvoll operationalisieren und somit auch empirisch nicht überprüfen. Pragmatisch hat sich daher mit der Zeit eine ,Sowohl-Als-auch“Position durchgesetzt. Es werden Instruktions- und Ermöglichungslernen zumeist kombiniert. Maier et al. (2014) argumentieren, es gelte, eine gute Balance aus Rahmung und Selbstexploration zu finden.

Die Charakterisierung von Training als bloßer Wissensvermittlung und stumpfer Verhaltensschulung greift unter der Prämisse von „Sowohl-als-auch“ von Instruktions- und Ermöglichungsdidaktik zu kurz. „Mit Personalentwicklungsmaßnahmen sollen Kompetenzen von Mitarbeitenden und Führungskräften, die zur Bewältigung beruflicher Situationen befähigen, erhalten, aufgebaut und weiterentwickelt werden. Kompetenzen werden benötigt, um selbstorganisiert und kreativ in offenen Situationen agieren zu können. (...) Sie sind multimodal, insofern sie kognitive, emotional-motivationale, volitive und aktionale Komponenten einschließen." (Kauffeld und Grote 2019, S. 170f.). Die Perspektive auf die Arbeitsbeziehung auf gemeinsamer Augenhöhe hat, das zeigen etliche Autoren (Alfermann und Stoll 2012; Jenkins 2017), längst auch in der Sportpsychologie Einzug gehalten.

Prominente Coaching-Positionierungen rekurrieren allerdings immer wieder mit Parolen wie „Hilfe zur Selbsthilfe“ (Rauen 2014) oder „Beratung ohne Ratschlag“ (Radatz 2008) einseitig auf das Konzept der Ermöglichungsdidaktik. Einmal abgesehen von der in sich paradoxen Struktur dieser Parolen, die auf den ersten Blick attraktiv wirken, lassen empirische Untersuchungen großen Zweifel an einer oft behaupteten, strengen ermöglichungsdidaktischen Orientierung im Coaching aufkommen. Deplazes et al. (2016) zeigen, dass Coaches überwiegend offene und geschlossene Fragen nutzen. Zirkuläre, hypothetische oder Skalierungsfragen werden lediglich im einstelligen Prozentbereich eingesetzt. Ermöglichungsdidaktik, so unsere Hypothese, scheint daher ein gern gepflegter Mythos im Coaching $\mathrm{zu}$ sein, aber offenbar nicht der Standard. $\mathrm{Zu}$ einer klaren Abgrenzung gegenüber Training vermag diese Unterscheidung folglich weniger beitragen (können) als es auf den ersten Blick erscheint.

\subsection{Experten- vs. Prozessberatung}

Reyer (2016) unterscheidet - wie schon Schein (2010) Beratungsformen, die die gemeinsame Auffassung einer symmetrischen Arbeitsbeziehung (Prozessberatung) teilen, von solchen, die eine asymmetrische Beziehung und Ressourcennutzung aufweisen (Expertenberatung). Zur ersten Gruppe zählt er u.a. Coaching, zur letzten Training. Mit dieser Differenzierung, die sich ebenfalls im sog. Funktionspendel (Wolff 2012) spiegelt, ließe sich Training von Coaching klar unterscheiden: 
- Die Expertenberatung tritt mit der Philosophie an, Klienten einen klaren Rat zu geben. Die Klienten kaufen Entscheidungswissen ein, über das sie selbst nicht verfügen. Berater verfügen über mehr Wissen und Erfahrung als ihre Kunden; das impliziert ein Hierarchiegefälle (typisch: Arzt, Anwalt sowie andere Professionen). Solche Berater haben oft eigene Interessen (Umsatz, Wieder- und Zusatzkauf, Weiterempfehlung, Reputation); Verkäufer sind zumeist vertraglich gebunden und nicht neutral.

- Die Prozessberatung unterstellt, dass Klienten selbst Experten sind und potenziell über alle relevanten Informationen verfügen oder diese recherchieren können. Berater maßen sich nicht an, es besser zu wissen als die Klienten, sie verhalten sich daher inhaltlich weitgehend abstinent. Aber sie konsultieren sie mit ihrem methodischen Wissen auf dem gemeinsamen Weg, eine gute Lösung zu finden. Sie zeigen Optionen auf, vermehren Perspektiven, reflektieren, simulieren - bewegen sich somit ,auf Augenhöhe" und geben die Entscheidung immer wieder an ihre Klienten zurück. Die Lösung wird in Koproduktion gefunden, Berater nehmen dabei die Funktion eines Katalysators ein. Eigene Interessen der Coaches sind nicht von der Hand zu weisen, es fehlt ihnen aber qua Rolle der verkäuferische Impetus. Daher ähneln Prozessberater eher neutralen Maklern.

Diese Differenzierung zweier Beratungsmodi erscheint zunächst ebenso attraktiv und überzeugend wie die zwischen Instruktions- und Ermöglichungsdidaktik. Bei näherer Betrachtung erweist sie sich jedoch als übertrieben polarisierend, wenn nicht sogar artifiziell. So unterscheidet das Modell des sog. Funktionspendels (Wolff 2012) drei Operationsbereiche (grün, gelb, rot). Man mag nun trefflich darüber streiten, wo genau die Grenzen zwischen diesen Bereichen verlaufen. Auch hier fällt eine mangelhafte Operationalisierung des Konzepts auf. Eine puristische Interpretation (Beratung ohne Ratschlag; Radatz 2008), würde argumentieren, dass Coaching ausschließlich im grünen Bereich statthaft sei. Das würde Coaches jedoch daran hindern, in Gänze wirksam zu werden. Klären, Reflektieren, Realitätsabgleich, Inspirieren, Umsetzen, Auswerten - all das setzt voraus, dass nicht nur Input von Klienten, sondern auch von Coaches kommt. Woran (Kriterien) soll etwas geklärt werden? Woher (von wem) kommt die Inspiration? Wie sonst sollte es zu Klärung und Inspiration kommen, wenn nicht in einer Koproduktion von Coach und Klient? Steht damit nicht das ganze Modell in seiner Ordnungsfunktion zur Disposition?

Fatzer, langjähriger Wegbegleiter des Vaters dieser Unterscheidung zwischen Experten- und Prozessberatung (Schein 2010), kommentiert: „Der reine Prozessberater ist eine didaktische Illusion der Beraterszene. Jeder Prozessberater ist längst auch Experte in Kulturentwicklung,
Führung, Karriere oder Strategieberatung“ (Fatzer 2009, S. 92).

Schmidt-Lellek und Fietze (2018) schließlich legen die Argumentation Schreyöggs (2010) dar, „dass sich anspruchsvolle Formen des Coachings nicht in einer reinen Prozessberatung erschöpfen können“, [weil] „die Prozessberatung je nach dem Kontrakt, je nach der Fragestellung, je nach dem Klienten und je nach der Situation des Klienten durch Sequenzen von Expertenberatung - und in manchen Fällen sogar durch das Arzt-Patient-Modell angereichert werden [muss]“ (Schreyögg 2010, S. 130). Die Autorin rät in einer späteren Publikation ,eine hohe Rollenvielfalt und Rollenvariabilität“" (Schreyögg 2015, S. 246) der Coaches an und erläutert diese auch an einem Fallbeispiel, in dem sie zwölf verschiedene Rollen einnimmt und plausibel argumentiert, warum und wie sie ,zwischen Direktivität und Non-Direktivität“" (Schreyögg 2015, S. 255) adaptiv wechselt.

Wie schon Fietze (2015) anklingen ließ, kann das Dilemma Experten- versus Prozessberatung in eine Synthese, einen dritten Modus der Beratung, aufgehoben werden, der spätestens seit der Veröffentlichung von Königswieser et al. (2006) Komplementärberatung genannt wird. Dieser Ansatz wurde eine Zeit lang vielfältig und konträr diskutiert, findet sich inzwischen aber nicht mehr im aktuellen Diskurs.

Strikker und Strikker (2011) entwickeln die Idee der Komplementärberatung weiter zum Komplementär-Coaching. Coaches dürfen sich nicht allein auf eine individuelle Unterstützung - symmetrisch, also auf Augenhöhe beschränken, so deren Argumentation. Die komplementäre Rolle müssten sie ebenfalls einnehmen können, insbesondere dann, wenn sie blinde Flecken bei Klienten hinsichtlich organisatorischer Konsequenzen wahrnähmen. Allerdings sei es die Pflicht der Coaches, prozessual aufmerksam zu beobachten, ob die Balance zwischen symmetrischer und komplementärer Kommunikation von Coaches und Klienten auch ausgewogen bleibe, raten die Autoren sibyllinisch an. Auch die zweite Argumentationsfigur, die zur Abgrenzung von Coaching und Training ins Feld geführt wird, versagt offensichtlich bzw. erweist sich als nicht trennscharf.

\subsection{Domänen und Kulturen}

Eine dritte Argumentationslinie verläuft entlang des Kulturkonzepts. Im Leistungssport steht der Wettbewerbsgedanke im Zentrum. Es geht darum, durch Leistung zum Sieg zu gelangen. Diese Funktion deckt sich nur partiell mit der in der Wirtschaftswelt. Es ist richtig, dass man Wirtschaft auch „sportlich“ (kriegerisch) betrachten kann. Daneben gibt es aber weitere Beschreibungen mit anderen Konnotationen, beispielsweise die Kooperation. Die Leistungserbringung in der Wirtschaft ist kein Selbstzweck, sondern zielt auch 


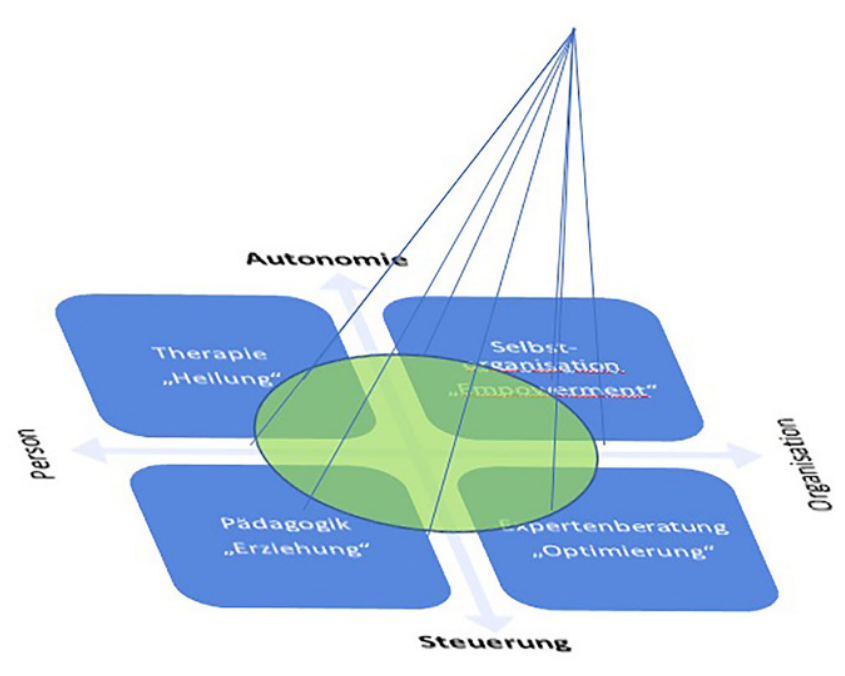

Abb. 3 Modifiziertes Funktionspendel. (Darstellung: T. Webers)

auf den Austauschprozess mit einer zahlenden Kundschaft. Während im Sport Leistung das „Spiel“ ist, hat man es in der Wirtschaft mit einer großen Bandbreite an „Spielen“ zu tun. Die Wettkampfarena und der Markt sind unterschiedliche Kontexte. Im Markt gilt nicht nur das Wettkampfprinzip, sondern auch Coopetition (Kunstwort: cooperation und competition). Man könnte - zurückgreifend auf den Beginn dieses Abschnitts - argumentieren, dass es sich bei Coaching und Training letztlich um zwei unvergleichbare Domänen handele. Ein Vergleich wäre folglich schlicht sinnlos.

Wenn sich die Perspektive auf Sport allerdings erweitert um Anwendungen in Prävention und Rehabilitation, im Betrieblichen Gesundheitsmanagement, aber auch im künstlerischen Bereich - und dies ist seit geraumer Zeit wahrnehmbar - verändert dies die Anforderungen an Trainer enorm. Neben den Leistungszielen rücken inzwischen weitere Ziele in den Vordergrund: z. B. Gesundheit, Fitness, Lifestyle, Spaß, Schönheit (Eitelkeit/Aussehen), Entspannung und Ausgleich, Naturerlebnis, materielle Gewinne, Freizeitgestaltung, Geselligkeit, Kontakte, Anschluss, Aggressionsabbau, sozialer Aufstieg, Prestige etc. Ob und wie sich der hier skizzierte Funktionswandel auf das Selbstverständnis von Trainern (und Coaches) auswirken wird, ist derzeit völlig offen. Die Wettkampfidee als Unterscheidungskriterium verliert so allerdings ihren Ausschließlichkeitscharakter. Der Vergleich von Coaching und Training produziert auf diese Weise wieder mehr Gemeinsamkeiten als Unterschiede.

Bachmann (2016) legt eine faktorenanalytisch fundierte Klassifizierung von Organisationen anhand ihres CoachingVerständnisses vor, die er anhand einer Stichprobe von Personalverantwortlichen aus Unternehmen und Institutionen in Deutschland $(N=96)$ gewann. Es zeigen sich vier Cluster, die sich aus der Kreuzung der beiden Dimensionen Autonomie vs. Steuerung sowie Person vs. Organisation ergeben:

- Therapie/Heilung (Autonomie/Person). Primär personenfokussiert, man möchte den Mitarbeitern etwas Gutes tun. Typische Themen sind: Work-Life-Balance, Zeitund Selbstmanagement. Die Ergebniserwartungen der Organisation sind eher unspezifisch und wenig formuliert.

- Selbstorganisation/Empowerment (Autonomie/Organisation). Primär organisationsfokussiert, man möchte die Zusammenarbeit verbessern. Typische Themen sind: Führung und Rollenklärung, Potenzialentwicklung. Es lässt sich eine gewisse Ergebnisoffenheit konstatieren.

- Expertenberatung/Optimierung (Steuerung/Organisation). Primär organisationsfokussiert, der „Laden soll laufen“. Typische Themen sind: Konflikte, Teamprobleme, Mikropolitik und Entscheidungen. Es herrscht eine deutliche Ergebniserwartung vor.

- Pädagogik/Erziehung (Steuerung/Person). Primär personenfokussiert, man möchte Defizite der Mitarbeiter bearbeiten. Typische Themen sind: Standing und Auftreten. Es herrscht eine deutliche Ergebniserwartung vor.

Bei genauerer Betrachtung fällt auf, dass sich diese vier Klassen mit dem sog. Funktionspendel (Wolff 2012) vergleichen lassen. Es wären zwei horizontale, vertikale sowie diagonale Lesarten darstellbar: Derart ließe sich beispielsweise (eindimensional) ein Kontinuum zwischen dem Pädagogen und dem Expertenberater ausdifferenzieren, indem ein gelber und ein grüner (neutraler) Bereich des Pendels postuliert würde. Solche Konzeptionierungen vermögen jedoch nicht zu überzeugen, weil mit diesen Varianten immer eine erklärungsbedürftige Hierarchie zwischen den einzelnen Klassen gesetzt würde und sich die Frage stellen würde, ab wann genau man vom einen Bereich in den anderen gelangt.

Logischer erschiene, das Pendel-Modell dreidimensional zu konzipieren, also über der Fläche der vier Klassen ausschlagen zu lassen. Damit würden die einzelnen Klassen jeweils von der Mitte aus zum Rand in grüne, gelbe und rote Bereiche unterteilt. Auf diese Weise würde impliziert, dass alle vier Quadranten in verschiedenen Ausprägungen gedacht und bewertet werden könnten, das Optimum, also der grüne Bereich, wäre dann eine Mischung aller vier Quadranten. Die ursprünglich im sog. Funktionspendel angelegt Wertung würde tendenziell abgeschwächt.

Mit der Adaption dieses Modells (s. Abb. 3) ließe sich nun nicht nur Coaching, sondern auch Training interpretieren. Es wäre vorstellbar, dass der Ausschlag des Pendels unterschiedliche Muster annehmen könnte. Auf diese Weise würden bspw. für Sport-Training die pädagogische Funktion sowie die Rolle der Führungskraft stärker betont. Für Coaching könnte eine stärkere Gewichtung der Funktionen 
„Therapie“ bzw. „Selbstorganisation“ dargestellt werden. All dies unter der Prämisse, dass prinzipiell alle vier Quadranten, wenn auch unterschiedlich stark, relevant wären und keiner prinzipiell ausgeschlossen werden dürfte. Damit wäre modelltheoretisch auf jeden Fall eine Schnittmenge zwischen Coaching und Training postuliert. Aber auch dieses Modell ließe sich letztlich nicht eindeutig operationalisieren. Diesen Nachteil teilt es mit allen Typenmodellen (bspw. in der Persönlichkeitspsychologie). Bachmann (2016) warnt zudem davor, Kulturen zu bewerten.

\section{Re-Professionalisierung}

Die Positionierungsbemühungen von Coaching, das konnte gezeigt werden, sind ambitioniert und werden mit erheblichem theoretischem Aufwand betrieben. Letztlich können sie aber nicht völlig überzeugen. Coaching und Training lassen sich zwar in einigen Aspekten unterscheiden, in anderen offenbaren sie andererseits Gemeinsamkeiten. Trennschärfe lässt sich nicht feststellen. So stellt sich die Frage, was ist mit einem Vergleich bzw. einer Abgrenzungsdiskussion gewonnen? Ein letzter Abschnitt kehrt daher zurück zur anfänglich dargestellten Professionalisierungsdiskussion und verfolgt das Ziel, potenziell neue, weiterführende Perspektiven $\mathrm{zu}$ eröffnen.

\subsection{Expertisierung vs. Professionalisierung}

Mieg (2018) gibt der Diskussion um Professionalisierung eine neue Ausrichtung. Seit bald hundert Jahren, so seine Argumentation, gibt es die Forschungstradition der Professionssoziologie. Doch ähnlich lange gibt es eine psychologische Expertenforschung. Beide Traditionen koexistieren, ohne dass es zu einem Austausch gekommen wäre. Im Gegenteil. So würde in der Soziologie fälschlicherweise Expertenarbeit oft mit Professionalisierung gleichgesetzt. In der Regel, so seine Begründung, gehe Professionalisierung mit Expertisierung einher, allerdings nicht umgekehrt. Die Fokussierung auf Expertisierung betrachtet damit weniger die Institutionenbildung als vielmehr die Entwicklung von Kompetenz.

Die psychologische Expertenforschung begann mit der Untersuchung von Schachspielern. Entgegen ursprünglichen Annahmen spielen jedoch generelle Fähigkeiten und Persönlichkeitsmerkmale (z.B.: Intelligenz), keine ausschlaggebende Rolle für die Entwicklung von Expertise. Vielmehr sind die Konzentration auf eine Domäne (Bereichsspezifität) und jahrelanges Training (Zehn-JahresRegel) entscheidend. „Expertise [stellt] eine hoch spezialisierte kognitive Anpassung an ein Problemfeld dar" (Mieg 2007, S. 65).
Diese Expertise ist im Alltag allgegenwärtig und produziert zwei interessante Phänomene: Wird in einem Diskurs jemand als Experte ausgewiesen, werden damit die anderen zu Laien erklärt. Doch solche Unterscheidungen sind spezifisch. Man kann auf einem Gebiet als Experte gelten, auf einem anderen zugleich als Laie. Die Expertenrolle ist lediglich relativ. ,,Experte“ ist letztlich eine Form von Interaktion, in deren Mittelpunkt die Attribution von Expertise auf eine Person, den Experten, steht“ (Mieg 2007, S. 67). Zu klären ist deshalb im Diskurs, ob die zum Experten erklärte Person tatsächlich über Sachwissen verfügt, das relevant für die Klärung des spezifischen Problems ist. Was zur Kompensation des Unsicherheitsgefühls beiträgt ist, dass das Wissen und Können, das der Experte anzubieten hat, grundsätzlich von jedem bereitgestellt werden könnte, wenn man denn selbst genügend Zeit hätte, es zu akkumulieren. Die Aspekte der Zeiteffizienz und die Bereichsspezifität charakterisieren die Expertise. An dieser Stelle eröffnen sich Übergänge zur Differenzierung von Amateur- und Profistatus.

Sowohl die Profession der Trainer als auch die der Coaches erweisen sich bei genauerer Betrachtung - im Vergleich zu klassischen Professionen (Arzt, Priester, Jurist etc.) - als unsicher institutionalisiert. Beide Professionen konkurrieren in der Praxis mit selbsternannten und/oder durch das Publikum autorisierte Experten. Hier sind es „Vorturner", die als Trainer auf die eigene athletische Biografie verweisen. Dort sind es „Menschenkenner“, die als Coaches bspw. mit der jahrelangen Erfahrung als Führungskraft werben.

Killing verweist auf weit verbreitete ehrenamtliche Tätigkeit und mangelnde Wertschätzung von ausgebildeten Trainern im Breitensport. Die Abschottung gegenüber Nicht-Ausgebildeten sei nicht ausreichend, der Neid gegenüber den gutverdienenden Profis wiederum dafür umso ausgeprägter. „Grundproblem der unzureichenden Professionalisierung der Trainer ist das nur teilprofessionalisierte Sportsystem, in dem haupt-, neben- und ehrenamtliche Funktionsträger mit ihren je eigenen Ressourcen und Wertesystemen aufeinander treffen" (Killing 2002, S. 52).

Auch in der Coaching-Szene werden die mangelnde soziale Schließung sowie die Zersplitterung der Verbände bedauert. Coaching sei lediglich ein Nebenberuf, wird schon seit vielen Jahren kritisiert (Middendorf 2004); und dass man Scharlatane nicht genügend aus dem Markt halten könne (Kühl 2008).

Beide Professionen werden nicht umhinkommen, den Wert einer Professionalisierung, zu der unter anderem eine Akademisierung sowie eine Institutionenbildung gehört, gegenüber der bloßen Expertisierung der Amateure zu behaupten, unter anderem mittels einer aktiven Öffentlichkeits- und Lobbyarbeit. 


\subsection{Die Konkurrenz der Professionen untereinander}

Professionelle Arbeit vollzieht sich, Mieg nimmt Bezug auf Abbott (1988), in einem Dreischritt: Diagnose, Inferenz, Maßnahme. Während Diagnosen (z.B.: Röntgenaufnahme) und Maßnahmen (z.B.: Physiotherapie) an Zulieferer oder Assistenten delegierbar sind, stellt die Inferenz, die Ableitung von Maßnahmen, das nicht delegierbare Herzstück professioneller Arbeit dar. Professionen schaffen Standards für Inferenz und kontrollieren diese. Das verschafft ihnen Autonomie. Allerdings dürfen Professionen nicht solitär betrachtet werden, sie konkurrieren im Feld mit anderen Professionen. Unterschiedliche Professionen deuten Sachverhalte unterschiedlich, bewerten also Diagnosen im Lichte ihrer eigenen Wissensbestände und erklärten Zuständigkeitsbereiche. Abbott hat hierfür den Begriff der Reduktion eingeführt.

Mieg (2018) führt exemplarisch aus, dass das Thema Umweltproblematik von verschiedenen Berufsgruppen reklamiert wird, die auf unterschiedliche Sachverhalte fokussieren, unterschiedliche Erklärungskonzepte liefern und unterschiedliche Maßnahmen vorschlagen. In seiner Darstellung lassen sich die Professionen in einem Feld, das durch die Dimensionen Problem-Reduktion und Formalisierung (Verfahrensstandardisierung, Routinen) aufgespannt wird, entsprechend lokalisieren: „Je mehr reduziert [Hervorhebung im Original], umso weniger hat die Tätigkeit mit Umwelt im engeren Sinn zu tun, z.B. kann die Bezeichnung ,Management' Tätigkeiten einbeziehen, die nichts mit Umwelt zu tun haben“" (Mieg 2018, S. 18). In einem solchen Tableau ließen sich Coaching und Training (sowie ggf. weitere Professionen) ebenfalls als spezifische Praktiken darstellen (s. Abb. 4), deren Spektrum sich jedoch überlappt, und man mag trefflich darüber streiten, ob die dargestellte Positionierung angemessen ist.

Die Ableitung von Maßnahmen (Inferenz) erfolgt als spezifische Antwort einer Profession auf eine Diagnose. Die Konkurrenz der Professionen kann, so Mieg (2018), auch als Zusammenspiel von mindestens vier Expertenrollen beschrieben werden:

1. Analysten bzw. Forscher, die nur für die diagnostischen Daten Gewährleistung übernehmen.

2. Formalexperten, die methodische Entscheidungsunterstützung leisten (Inferenz).

3. Relative Experten, die nur Informationen beschaffen.

4. Professionelle, die für die ganze Kette der Tätigkeiten verantwortlich zeichnen.

Das spezifische Wissen, auf das sich der Professionelle bezieht, darf allerdings nicht statisch verstanden werden. Vielmehr sind „Professionen (...) wissens- und erkenntnisgestützte Berufe" (Mieg 2018, S. 16). Damit vollzieht sich ein Wechsel der Perspektive hin zur Prozesshaftigkeit.

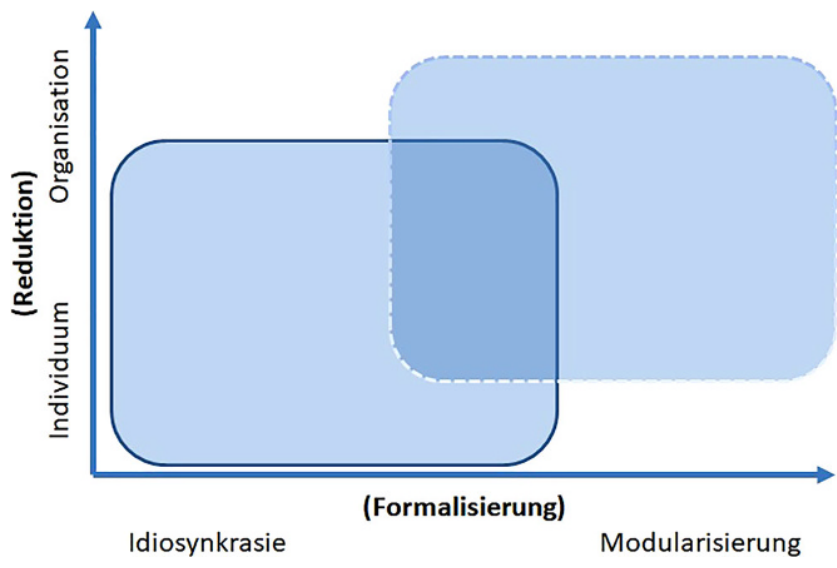

Abb. 4 Vereinfachte Darstellung des Tätigkeitsfeldes im Bereich professionalisierte Dienstleistung

Zugleich ist Inferenz keine schlichte Exekution von Wissen, sondern berücksichtigt Kontextbedingungen. Sie bezieht auch Werte mit ein. Mit anderen Worten: Der Professionelle erbringt eine persönliche Dienstleistung. Anders als im Handwerk produziert er kein Produkt, sondern er erklärt, macht begreifbar, stiftet Sinn. „Professionelle Arbeit arbeitet wesentlich mit an einem rationalen System der Welterklärung“ (Mieg 2018, S. 24).

Die Konkurrenz der Professionen untereinander lässt sich prinzipiell nicht auflösen. Das Bundesinstitut für berufliche Bildung (BIBB) ist nicht zuständig und die Entwicklung nach der „Bologna-Reform“ zeigt, dass alte Definitionen zunehmend in Fluss geraten - was die einen erfreut und die anderen bedauern. Inzwischen lässt sich der klare Trend beobachten, dass sich Supervisions- und Trainerverbände zunehmend als Coaching-Verbände positionieren: der ursprünglich als Verkaufstrainerverband gegründete „Berufsverband der Trainer, Berater und Coaches“ - BDVT (2008), die bislang auf Supervision fokussierte „European Association for Supervision and Coaching“ EASC (2011) und der schweizerische „Berufsverband für Coaching, Supervision und Organisationsberatung “ - bso (2011) sowie die sich traditionell ebenfalls als eindeutige Heimat der Supervisoren verstehende „Deutsche Gesellschaft für Supervision und Coaching“" DGSv (2016). Die mit der Gründung des Roundtable der Coachingverbände (RTC) beabsichtige Marktschließung erscheint - nach der Veröffentlichung eines gemeinsamen Papiers (RTC 2015) - zum Stillstand gekommen zu sein. Dem Markt lässt sich bislang keine klare Abgrenzung der Profession Coaching vermitteln. Dies stellt ein gravierender Nachteil in der Marktbearbeitung dar. 


\subsection{Professionen als Dienstleister}

Mieg (2018) führt die Charakterisierung der professionalisierten Arbeit als Dienstleistung nicht weiter aus. Doch ergeben sich dadurch weitere Einsichten. So benennt Greif fünf Aspekte, die Coaching als Dienstleistung ausmachen:

- „sehr hohe Intangibilität,

- Untrennbarkeit (Uno-actu-Prinzip) und Ko-Kreation der Leistung,

- hohe Heterogenität (Berücksichtigung der Individualität der Klienten),

- strikte Vertraulichkeit und

- relative Unabhängigkeit von Auftraggeber/Kostenträger und Dienstleistungsnehmer" (Greif 2015, S. 62)

Weder im Coaching, noch im Training wird ein Produkt im klassischen Sinne hergestellt. Eine neue Perspektive auf ein berufliches Problem oder eine taktische Spielvariante im Sport kann man nicht anfassen, lagern, weiterverkaufen. Das Bild des Nürnberger Trichters (Instruktionsdidaktik) erweist sich als unpassend. Coaching und Training sind untrennbar von der Interaktion, in der Ko-Kreation erweisen sie sich als sinnvoll. Eine passive Konsumentenhaltung nutzt Coaching-Klienten wie Athleten nichts. Sie sind keine Verbraucher, sondern Koproduzenten. Sie bringen eigene Kompetenz und Engagement in den Prozess mit ein. Wenn sie dies unterlassen, kann weder Coaching noch Training fruchten.

Mieg (2010), der die Frage der Professionalisierung von Mediation diskutiert, argumentiert auch auf der Ebene der Klienten mit dem Konzept der Autonomieregulation. Wenn die Eigenverantwortlichkeit des Klienten ernst genommen wird, ergibt sich - in den Worten Schreyöggs (2015) - eine Bandbreite an Direktivität. Der Mediator erscheint einerseits als „Nullstelle“, andererseits als „Manager“ und "Regisseur". Mieg, der die Veröffentlichung von Maiwald (2003) zur Professionalisierung der Familienmediation referiert, beschreibt Mediation daher als ,paradoxales Professionalisierungsprojekt". Diese grundsätzliche Paradoxie liegt ebenfalls Training und Coaching zugrunde. Dies lässt sich, das hatten wir schon diskutiert, an den Konzepten Instruktions- vs. Ermöglichungsdidaktik bzw. Experten- vs. Prozessberatung beobachten. Letztlich sind diese Konzepte nicht disjunktiv.

Sowohl Training als auch Coaching müssen hoch adaptiv auf die Individualität der Klienten reagieren. Hier könnte man begründet argumentieren, dass der Grad der Individualisierung im Coaching insgesamt höher ist als im Training. Denn das thematische Design ist im Training zumeist eingeschränkt (Sportart). Viele Trainings finden im Gruppenmodus statt. Wollte man diese Unterscheidung allerdings trennscharf machen, müsste man Gruppen- und Team-Coaching aus dem Coaching-Repertoire ausschließen. In der Sport- domäne müsste man ein Einzeltraining oder die Wettkampfunterstützung fortan Coaching nennen. Es ist leicht ersichtlich, dass solche apodiktischen Unterscheidungen nicht nur artifiziell wirken, sondern auch wenig praktikabel erscheinen. Daher kann der Hinweis auf graduelle Unterschiede lediglich als Behauptung geführt werden. Ähnlich verhält es sich bei den Anforderungen an Vertraulichkeit (bspw. der Coach diskret im Hintergrund, der Trainer als „Pressesprecher" des Teams) als auch bei der organisationalen Eingebundenheit (externer vs. interner Coach).

Weitere Schwierigkeiten kommen hinzu. Wenn Coaching und Training nicht Produkte sein können, sondern prinzipiell Dienstleistungen sind, ergibt sich eine Erklärungsbedarf gegenüber dem Markt (Nerdinger 2018). Produkte sind über Anforderungskataloge (Gewicht, Farbe, Features, Mindesthaltbarkeitsdatum) leicht vergleichbar. Die Qualität von Dienstleistungen nicht. Kunden müssen Dienstleistern vertrauen, sich auf ihre Erfahrung oder Empfehlungen verlassen. Dies kann als Markthürde wirken. In der Literatur wird die Agency-Theorie als Erklärungsansatz diskutiert. Diese Theorie fokussiert auf Informationsasymmetrien zwischen Leistungserbringern und Nachfragern. Stephan und Groß beschreiben dies als „Gefahr für die Nachfrageseite, einen Coaching-Anbieter mit mangelnden Qualifikationen (,Hidden Characteristics'), falschen Absichten (,Hidden Intentions') oder verdeckten Motivationsdefiziten zu akquirieren" (Stephan und Groß 2011, S. 14). Zur Problemlösung empfiehlt die Theorie ScreeningMaßnahmen für den Nachfrager und Signalling-Maßnahmen für den Anbieter. Selbstmarketing erweist sich bei wissensintensiven Dienstleistungen als unabdingbar notwendiger Aspekt der professionellen Arbeit. Doch womit soll man inhaltlich argumentieren, wenn sich doch alle Unterscheidungsfiguren, die wir bislang diskutiert haben, als nicht trennscharf erwiesen haben?

\subsection{Qualitätsmanagement}

Wenn die Inferenz, als Ableitung von Maßnahmen, das nicht delegierbare Herzstück jeder professionellen Arbeit ist, ergibt sich damit eine weitere Argumentationsfigur: Ein Ermessensspielraum (Freidson 2001). Weil dieser relativ ist, kann er nicht nur individuell zugemessen werden. Professionelle sind auf die Diskussion um Best Practice innerhalb der „epistemischen Gemeinschaft“ (Mieg 2018, S. $27 \mathrm{f}$ ), ob es sich um Berufsverbände oder informelle Netzwerke handelt, angewiesen. Lege artis ist etwas Dynamisches und Soziales.

Ob nun als Verbandsarbeit und/oder als Supervision, der Austausch und die Beratung in der Profession (Scientific Community) leisten einen Mehrwert zur professionellen Arbeit des einzelnen Professionellen. Dies unterscheidet die Professionellen eben auch von den Experten. Daher gehört 
der Hinweis darauf generell ins Marketingkonzept der Professionen.

\section{Fazit}

Die Abgrenzungsdiskussion der Coaching- gegenüber der Sport-Domäne ist, so konnten wir zeigen, in weiten Strecken wenig sinnvoll. Es lassen sich etliche Gemeinsamkeiten sowie Unterschiede benennen. Offenbar gibt es attraktive Aspekte der Nutzung der Sportmetapher, aber gleichfalls hinderliche Konnotationen. Die üblicherweise diskutierten Unterscheidungskriterien sind in der Regel nicht trennscharf. Vergleiche hinken, sagt der Volksmund.

Sowohl in der Sport-, als auch in der Business-Domäne erscheinen die Professionen der Trainer und Coaches unsicher professionalisiert. Es wird daher kein Weg daran vorbeiführen, die Professionalisierung weiter zu forcieren: Sowohl im Wettbewerb durch Positionierung, als auch durch Institutionalisierung. Wenn Fietze (2015) einen allgemeinen Entberuflichungsprozess (De-Professionalisierung) in der Moderne diagnostiziert, kann es für die Coaching-Branche wenig sinnvoll sein, hinter dem Ideal alter Professionen herzulaufen, wenn sich diese gerade auflösen/relativieren. Doch wäre die Orientierung am Modell der Professionalisierung im weiteren Sinne - also am Markt alleine - ebenfalls nicht zweckdienlich.

Professionalisierung von Coaching kann aber über einen dritten Weg zwischen Staat und Markt gelingen. Wenn auch theoretisch die Abgrenzungsdebatte nicht trennscharf $\mathrm{zu}$ entscheiden ist, zählt doch in der Praxis viel mehr die Akzeptanz der Dienstleistung Coaching beim Publikum. Paradoxe Parolen wie Hilfe zur Selbsthilfe scheinen diese zu befördern.

„Das Gemeinsame aller Professionen ist die Definitionsmacht hinsichtlich der Leistungsbewertung. Professionen schaffen Standards der Leistungsbewertung und kontrollieren diese. Dies ist der abstrakte Mehrwert von Profession gegenüber bloßem Expertentum“ (Mieg 2018, S. 33). Die referierten Erkenntnisse der Professionalisierungsdiskussion verweisen daher auf unabdingbare Handlungsmaxime: Abgrenzung nicht nur durch spezifisches Wissen (Weiterbildung), sondern ebenfalls durch einen Ethikkodex sowie eine starke, auch exklusive verbandliche Selbstorganisation. Der Mehrwert der Profession erschließt sich zudem durch ein überzeugendes Qualitätsmanagement (inkl. Supervision) und eine aktive Öffentlichkeitsarbeit. Das Ziel lautet: eine stabile positive Kompetenz-Attribution zu etablieren. Professionalisierung darf deshalb nicht als statischer $\mathrm{Zu}$ stand, sondern muss als Prozess (Aufgabe) begriffen werden.
Interessenkonflikt T. Webers und R. Zickermann geben an, dass kein Interessenkonflikt besteht.

Open Access Dieser Artikel wird unter der Creative Commons Namensnennung 4.0 International Lizenz (http://creativecommons.org/ licenses/by/4.0/deed.de) veröffentlicht, welche die Nutzung, Vervielfältigung, Bearbeitung, Verbreitung und Wiedergabe in jeglichem Medium und Format erlaubt, sofern Sie den/die ursprünglichen Autor(en) und die Quelle ordnungsgemäß nennen, einen Link zur Creative Commons Lizenz beifügen und angeben, ob Änderungen vorgenommen wurden.

\section{Literatur}

Abbott, A. (1988). The system of professions. Chicago: University of Chicago Press.

Alfermann, D., \& Stoll, O. (2012). Sportpsychologie: Ein Lehrbuch in 12 Lektionen (4. Aufl.). Aachen: Meyer \& Meyer Sport.

Anger, J., \& Schober, V. (2014). Rechtliche Rahmenbedingungen von Supervision und Coaching in Europa. Wien: ANSE.

Arnold, R. (2012). Ermöglichungsdidaktik - die notwendige Rahmung einer nachhaltigen Kompetenzreifung. Berufsbildung in Wissenschaft und Praxis, 41(2), 45-48.

Bachmann, T. (2016). Implizite Theorien über Lernen und Veränderungen durch Coaching in Organisationen - Eine empirische Untersuchung. Organisationsberatung - Supervision - Coaching, 23(3), 231-253. https://doi.org/10.1007/s11613-016-0467-z.

Baumann, S. (2015). Psychologie im Sport (6. Aufl.). Aachen: Meyer $\&$ Meyer Sport.

Bechthold, A. (2014). Coaching aus Trainersicht. Leistungssport, 44(2), 22-26.

Birgmeier, B. (2006). Coaching als Methode und/oder Profession? Organisationsberatung - Supervision - Coaching, 13(1), 19-30. https://doi.org/10.1007/s11613-006-0003-7.

Brand, R. (2010). Sportpsychologie. Wiesbaden: Springer.

Chelladurai, P., \& Riemer, H. A. (1998). Measurement of leadership in sport. In J.L. Duda (Hrsg.), Advances in sport and exercise psychology measurement (S. 227-253). Morgantown: Fitness Information Technology.

Cushion, C., \& Lyle, J. (2016). Conceptualising Sport-Coaching: Some Key Questions and Issues. In R. Wegener, M. Loebbert \& A. Fritze (Hrsg.), Coaching-Praxisfelder. Forschung und Praxis im Dia$\log$ (Bd. 2, S. 117-134). Wiesbaden: Springer.

Deplazes, S., Schwyter, S., \& Möller, H. (2016). Ein Blick auf Coachingprozesse - Die Interventionen des Coachs, dargestellt und analysiert mittels des KaSyCo-C. Coaching Theorie \& Praxis, 2(1), 9-19. https://doi.org/10.1365/s40896-016-0010-4.

Eberspächer, H. (2005). Was Coaching von der Sportpsychologie lernen kann. Wirtschaftspsychologie aktuell, 12(1), 27-28.

Fatzer, G. (2009). Perspektiven. Organisationsentwicklung, 28(2), 92.

Fietze, B. (2015). Coaching auf dem Weg zur Profession? Eine professionssoziologische Einordnung. In A. Schreyögg \& C. SchmidtLellek (Hrsg.), Die Professionalisierung von Coaching. Ein Lesebuch für den Coach (S. 3-21). Wiesbaden: Springer.

Freidson, E. (2001). Professionalism: The third logic. Cambridge: Polity.

Greif, S. (2015). Evaluation von Coaching: Eine schwer zu bewertende Dienstleistung. In A. Schreyögg \& C. Schmidt-Lellek (Hrsg.), Die Professionalisierung von Coaching. Ein Lesebuch für den Coach (S. 47-69). Wiesbaden: Springer.

Greif, S., Möller, H., \& Scholl, W. (2018). Coachingdefinitionen und -konzepte. In S. Greif, H. Möller \& W. Scholl (Hrsg.), Handbuch Schlüsselkonzepte im Coaching (S. 1-9). Berlin: Springer.

Griffo, J.M., Jensen, M., Anthony, C.C., Baghurst, T., \& Hodges Kulinna, P. (2019). A decade of research literature in sport coaching (2005-2015). International Journal of Sports 
Science \& Coaching, 14(2), 205-215. https://doi.org/10.1177/ 1747954118825058.

Hänsel, F., Baumgärtner, S. D., Kornmann, J., \& Ennigkeit, F. (2016). Sportpsychologie. Heidelberg: Springer.

Hohmann, A., Lames, M., \& Letzelter, M. (Hrsg.). (2014). Einführung in die Trainingswissenschaft (6. Aufl.). Wiebelsheim: Limpert.

Hottenrott, K. (2017). Potenziale der Individualisierung im Sport und Gesundheitscoaching stärker nutzen. Deutsche Zeitschrift für Sportmedizin, 68(9), 187-188. https://doi.org/10.5960/dzsm. 2017.294.

ICF (o.J.). How is coaching distinct from other service professions? www.coachfederation.org/faqs. Zugegriffen: 30. Aug. 2019.

Ives, Y. (2008). What is coaching - an exploration of conflicting paradigms. International Journal of Evidence Based Coaching and Mentoring, 6(2), 100-113. https://doi.org/10.24384/IJEBCM/6/2.

Jenkins, S. (2017). The deconstruction and reconstruction of sports coaching: An interview with Professor Robyn Jones. International Journal of Sports Science \& Coaching, 12(4), 413-420. https://doi.org/10.1177/1747954117718019.

Jowett, S., \& Cockerill, I. M. (2003). Olympic medallists' perspective of the athlete-coach relationship. Psychology of Sport and Exercise, 4(4), 313-331. https://doi.org/10.1016/S14690292(02)00011-0.

Kauffeld, S., \& Grote, S. (2019). Personalentwicklung. In S. Kauffeld (Hrsg.), Arbeits-, Organisations- und Personalpsychologie für Bachelor (3. Aufl. S. 167-210). Heidelberg: Springer.

Killing, W. (2002). Der Trainerberuf in der Krise. Leistungssport, $32(2), 50-55$.

Königswieser, R., Sonuc, E., \& Gebhardt, J. (Hrsg.). (2006). Komplementärberatung. Das Zusammenspiel von Fach- und ProzeßKnow-how. Stuttgart: Klett.

Kühl, S. (2008). Die Professionalisierung der Professionalisierer? Das Scharlatanerieproblem im Coaching und der Supervision und die Konflikte um die Professionsbildung. Organisationsberatung Supervision - Coaching, 15(3), 260-294. https://doi.org/10.1007/ s11613-008-0085-5.

Leder, A. (2007). Führen oder coachen? Beides sollte nicht verwechselt werden. Der Chef als Coach. wirtschaft+weiterbildung, 18(9), 52-56.

Maier, G.W., Sobiraj, S., Steinmann, B., \& Nübold, A. (2014). Personalentwicklung I: Training und Transfer. In H. Schuler \& K. Moser (Hrsg.), Lehrbuch Organisationspsychologie (Bd. 5, S. 127-175). Bern: Huber.

Maiwald, K.-O. (2003). Der unsichtbare Mediator: Probleme der Ausweisung beruflicher Leistung in der Familienmediation. In H. A. Mieg \& M. Pfadenhauer (Hrsg.), Professionelle Leistung Professional Performance: Positionen der Professionssoziologie (S. 195-226). Konstanz: UVK.

Middendorf, J. (2004). Die wirtschaftliche Situation von Coachs - Follow-up. Wirtschaftspsychologie aktuell, 11(1), 13-15.

Mieg, H. (2007). Expertisierung vs. Professionalisierung. Relative und andere Experten aus Sicht der psychologischen Expertiseforschung. In H. Mieg (Hrsg.), Professionalisierung. Essays zu Expertentum, Verberuflichung und professionellem Handeln (S. 61-72). Potsdam: Verlag der Fachhochschule. 2018.

Mieg, H. (2010). Professionalisierung. Eine dreifache Autonomieregulation. In H. Mieg (Hrsg.), Professionalisierung. Essays zu Expertentum, Verberuflichung und professionellem Handeln (S. 47-59). Potsdam: Verlag der Fachhochschule. 2018.

Mieg, H. (2018). Professionalisierung - eine konzeptionelle Wiederbelebung. In H. Mieg (Hrsg.), Professionalisierung. Essays zu Expertentum, Verberuflichung und professionellem Handeln (S. 11-36). Potsdam: Verlag der Fachhochschule.

Nerdinger, F. (2018). Qualität der Dienstleistung ,Coaching'. In S. Greif, H. Möller \& W. Scholl (Hrsg.), Handbuch Schlüsselkonzepte im Coaching (S. 475-483). Heidelberg: Springer.

North, J. (2017). Sport coaching research and practice. Ontology, Interdisciplinarity and critical realism. London: Routledge.
Radatz, S. (2008). Beratung ohne Ratschlag. Systemisches Coaching für Führungskräfte und BeraterInnen. Wien: Institut für systemisches Coaching und Training.

Rauen, C. (2014). Coaching (3. Aufl.). Göttingen: Hogrefe.

Reyer, T. (2016). Beratungsdschungel? Beratung, Coaching, Therapie, Supervision und mehr Differenzierung von Arbeitsformaten der Prozessberatung. Organisationsberatung - Supervision Coaching, 23(4), 463-473. https://doi.org/10.1007/s11613-0160478-9.

Roth, G., \& Ryba, A. (2016). Coaching, Beratung und Gehirn. Neurobiologische Grundlagen wirksamer Veränderungskonzepte. Stuttgart: Klett-Cotta.

RTC (2015). Profession: Coach. Ein Commitment des Roundtable der Coachingverbände. https://www.roundtable-coaching.eu/wpcontent/uploads/2015/03/RTC-Profession-Coach-2015-03-19Positionspapier.pdf. Zugegriffen: 16. März 2019.

Schaper, N., \& Sonntag, K. (2007). Weiterbildungsverhalten. In D. Frey \& L. von Rosenstiel (Hrsg.), Wirtschaftspsychologie. Enzyklopädie der Psychologie, (Bd. D/III/6, S. 573-648). Göttingen: Hogrefe.

Schein, E. (2010). Prozessberatung für die Organisation der Zukunft: Der Aufbau einer helfenden Beziehung (3. Aufl.). Bergisch-Gladbach: EHP.

Schlicht, W. (2008). Sportpsychologie - Eine Standortsuche. In W. Schlicht \& B. Strauß (Hrsg.), Grundlagen der Sportpsychologie. Enzyklopädie der Psychologie, (Bd. D/V/1, S. 1-31). Göttingen: Hogrefe.

Schmidt-Lellek, C. (2006). Anmerkungen zur Professionalisierung des Coaching auf dem Hintergrund des klassischen Professionsbegriffs. Organisationsberatung - Supervision - Coaching, 13(2), 183-192. https://doi.org/10.1007/s11613-006-0025-1.

Schmidt-Lellek, C., \& Fietze, B. (2018). Professionalisierung im Coaching. In S. Greif, H. Möller \& W. Scholl (Hrsg.), Handbuch Schlüsselkonzepte im Coaching (S. 457-465). Berlin: Springer.

Schnabel, G., Harre, H.-D., \& Krug, J. (Hrsg.). (2011). Trainingslehre - Trainingswissenschaft: Leistung-Training-Wettkampf (2. Aufl.). Aachen: Meyer \& Meyer Sport.

Schreyögg, A. (2010). Ist Coaching reine Prozessberatung oder sind hier auch andere Beratungsmodelle relevant? Organisationsberatung - Supervision - Coaching, 17(2), 119-132. https://doi.org/ 10.1007/s11613-010-0185-x.

Schreyögg, A. (2012). Abgrenzung zu anderen Beratungsformaten. In DBVC (Hrsg.), Leitlinien und Empfehlungen für die Entwicklung von Coaching als Profession. Kompendium mit den Professionsstandards des DBVC (S. 26-29). Osnabrück: DBVC.

Schreyögg, A. (2015). Die potenzielle Rollenvielfalt des Coachs. In A. Schreyögg \& C. Schmidt-Lellek (Hrsg.), Die Professionalisierung von Coaching. Ein Lesebuch für den Coach (S. 245-256). Wiesbaden: Springer.

Schulz, F. (2016). Ein organisationstheoretischer Ansatz zur Erforschung diskursiver Prozesse im Management Coaching. Coaching Theorie \& Praxis, 2(1), 1-7. https://doi.org/10.1365/ s40896-016-0009-x.

Smith, R.E., \& Smoll, F.L. (1996). The coach as focus of research and intervention in youth sport. In F.L. Smoll \& R.E. Smith (Hrsg.), Children and youth in sport. A biopsychosocial perspective (S. 125-141). Madison Dubuque: Brown \& Benchmark.

Stephan, M., \& Groß, P.P. (2011). Coaching aus wirtschaftswissenschaftlicher Sicht - Ergebnisse der Marburger Coaching Studie 2009. In M. Stephan \& P.-P. Gross (Hrsg.), Organisation und Marketing von Coaching (S. 3-34). Wiesbaden: VS.

Strikker, H., \& Strikker, F. (2011). Komplementär-Coaching: Herausforderungen an Coaching im Change und in der Krise. Eine theoretische Annäherung. In B. Birgmeier (Hrsg.), Coachingwissen (2. Aufl. S. 339-353). Wiesbaden: VS.

Weibler, J. (2013). Entzauberung der Führungsmythen. München: Roman-Herzog-Institut. 
Weineck, J. (2019). Optimales Training (17. Aufl.). Balingen: Spitta $\mathrm{GmbH}$.

Wolff, U. (2012). Anwendungsbereiche. In DBVC (Hrsg.), Leitlinien und Empfehlungen für die Entwicklung von Coaching als Profession. Kompendium mit den Professionsstandards des DBVC (S. 21-26). Osnabrück: DBVC.

Publisher's Note Springer Nature remains neutral with regard to jurisdictional claims in published maps and institutional affiliations. 